\title{
The Energetics of the Break-up of a Sheet and of a Rivulet on a Vertical Substrate in the Presence of a Uniform Surface Shear
}

\section{Stress}

\author{
S. K. WILSON, J. M. SULLIVAN AND B. R. DUFFY
}

Department of Mathematics and Statistics, University of Strathclyde, Livingstone Tower, 26 Richmond Street, Glasgow G1 1XH, United Kingdom

(Received 17th November 2008, revised 18th May, 10th November and 17th December 2010)

We use the lubrication approximation to obtain a complete description of the energetics of the break-up both of a fluid sheet of uniform thickness into a periodic array of infinitely many identical thin rivulets and of a single thin rivulet into one or more identical subrivulets on a vertical substrate in the presence of a prescribed uniform longitudinal shear stress at the free surface of the fluid by comparing the total energies of the different states. For both problems the situation when the volume flux is positive is relatively straightforward (and, in particular, qualitatively the same as that in the case of no prescribed shear stress), but when the volume flux is negative it is more complicated. However, whatever the value of the prescribed shear stress, there is always a critical thickness below which it is energetically favourable for a sheet to break up into rivulets and a critical semi-width below which it is energetically favourable for a rivulet to remain as a single rivulet, and a critical thickness above which it is energetically favourable for a sheet to remain as a sheet and a critical semi-width above which it is energetically favourable for a rivulet to break up into sub-rivulets. 


\section{Introduction}

There are many physical situations that involve sheets and/or rivulets of fluid subject to significant pressure and/or shear forces due to an external airflow; examples include various coating flows, flows of condensate within heat exchangers, and even the flow of rainwater on the cables of cable-stayed bridges which is believed to play a key role in the so-called "Rain-Wind-Induced Vibrations" that such cables can undergo on wet and windy days (see, for example, Robertson et al. (2010)). As a consequence there is an enduring theoretical and experimental interest in the possible break-up of a sheet of fluid into rivulets and the possible break-up of a rivulet into sub-rivulets in the presence of an external airflow.

The energetics of the break-up of a sheet of fluid into rivulets and the break-up of a rivulet into sub-rivulets have been studied by many previous authors, including Hartley and Murgatroyd (1964), Hobler (1964), Bankoff (1971), Mikielewicz \& Moszynski (1976), Mikielewicz \& Moszynski (1978), Chung \& Bankoff (1980), Doniec (1988), Doniec (1991), Schmuki \& Laso (1990), Hughes \& Bott (1998), El-Genk \& Saber (2001), El-Genk \& Saber (2002), Saber \& El-Genk (2004), Myers, Liang \& Wetton (2004), Wilson \& Duffy (2005a), Wilson \& Duffy (2005b) and Sullivan, Wilson \& Duffy (2008). In particular, in their landmark study building on the pioneering work by Hobler (1964) and Bankoff (1971), Mikielewicz \& Moszynski (1976) analysed the critical condition for the break-up of a sheet of uniform thickness draining under gravity down a vertical substrate into a periodic array of identical rivulets. In particular, by equating the energies of the two states and minimising the energy of the rivulet state per unit width of the sheet, they determined the critical thickness above which it is energetically favourable for the sheet 
to remain as a sheet but below which it is energetically favourable for it to break up into rivulets. Adoping a similar approach, Schmuki \& Laso (1990) analysed the break-up of a rivulet draining under gravity down a vertical or an inclined substrate into several identical sub-rivulets by comparing the energies of the two states. El-Genk \& Saber (2001) minimised the energy of a rivulet draining under gravity down a vertical substrate and hence obtained an expression for the critical thickness of a uniform sheet. Subsequently, the same approach was used by El-Genk \& Saber (2002) to analyse flow of an evaporating fluid on a uniformly heated vertical substrate and by Saber \& El-Genk (2004) to analyse flow on an inclined substrate in the presence of a prescribed non-uniform shear stress. Myers, Liang \& Wetton (2004) investigated the break-up of a rivulet on an inclined substrate in the presence of a prescribed uniform longitudinal shear stress at its free surface into two sub-rivulets both numerically and asymptotically in the thin-film limit. Specifically, by comparing the energies of states with one rivulet and two (in general, non-identical) sub-rivulets, they calculated some examples of the critical width below which it is energetically favourable for a purely gravity-driven rivulet to remain as a single rivulet but above which it is energetically favourable for it to break up into two sub-rivulets. In addition, they conjectured that it is never energetically favourable for a purely shear-stress-driven rivulet to break up into two sub-rivulets. Wilson \& Duffy (2005b) calculated when it is energetically favourable for a thin rivulet on a vertical substrate in the presence of a prescribed uniform longitudinal shear stress to break up into two (again, in general, unequal) sub-rivulets. In particular, they found that when break-up occurs the most energetically favourable state is that of two identical subrivulets, and disproved the conjecture of Myers, Liang \& Wetton (2004) by showing that it can be energetically favourable for a purely shear-stress-driven rivulet to break up into two identical sub-rivulets. An analysis of the break-up of a thin rivulet of perfectly 
wetting fluid (i.e. a rivulet with zero contact angle) on an inclined substrate into several identical sub-rivulets was performed by Wilson \& Duffy (2005a) (for purely gravitydriven rivulets) and by Sullivan, Wilson \& Duffy (2008) (for rivulets in the presence of a prescribed uniform longitudinal shear stress).

In the present work we use the lubrication approximation to obtain a complete description of the energetics of the break-up both of a sheet of uniform thickness into a periodic array of infinitely many identical thin rivulets and of a single thin rivulet into one or more identical sub-rivulets of a non-perfectly-wetting fluid on a vertical substrate in the presence of a prescribed uniform longitudinal shear stress at the free surface of the fluid by comparing the total energies of the different states.

\section{Solution for a Rivulet and for a Uniform Sheet}

\subsection{Solution for a Rivulet}

Wilson \& Duffy (2005b) obtained the solution for the steady unidirectional flow of a thin symmetric rivulet of Newtonian fluid with constant semi-width $a$ and constant volume flux $Q$ on a vertical substrate subject to a prescribed uniform longitudinal shear stress $\tau$ at its free surface, where positive values of $\tau$ correspond to the shear stress acting downwards (i.e. gravity and shear-stress effects co-operate) and negative values to it acting upwards (i.e. gravity and shear-stress effects compete). We employ Cartesian axes Oxyz with the $x$ axis vertically downwards, the $y$ axis horizontal and the $z$ axis perpendicular to the substrate $z=0$, and non-dimensionalise in the natural way using the capillary length $\ell=(\gamma / \rho g)^{1 / 2}$ to non-dimensionalise length in the $y$ direction, the characteristic rivulet thickness $\beta \ell$ to non-dimensionalise length in the $z$ direction, the characteristic velocity $U=\rho g \beta^{2} \ell^{2} / \mu$ to non-dimensionalise velocity in the $x$ direction, the characteristic pressure $\rho g \beta \ell$ to non-dimensionalise variations in pressure, and the characteristic stress 
$\rho g \beta \ell$ to non-dimensionalise the prescribed shear stress, where the constants $\gamma, \rho$ and $\mu$ denote the surface tension, density and viscosity of the fluid, respectively, the constant $g$ denotes acceleration due to gravity, and the constant $\beta(0<\beta \ll 1)$ is the (non-zero) static contact angle. The velocity $\mathbf{u}=u(y, z) \mathbf{i}$, pressure $p(y, z)$ and free surface profile $z=h(y)$ then satisfy $0=1+u_{z z}, 0=p_{y}$ and $0=p_{z}$ subject to $p=-h^{\prime \prime}$ and $u_{z}=\tau$ on $z=h, u=0$ on $z=0$, and $h=0$ and $h^{\prime}=\mp 1$ at $y= \pm a$ (where a prime denotes differentiation with respect to argument), and hence are given by

$$
u=\frac{(2 h-z) z}{2}+\tau z, \quad p=\frac{1}{a}, \quad h=\frac{a^{2}-y^{2}}{2 a} .
$$

The volume flux down the rivulet (which may be positive or negative depending on the relative strengths of gravity and shear-stress effects) is therefore given by

$$
Q=\int_{-a}^{+a} \int_{0}^{h(y)} u(y, z) \mathrm{d} z \mathrm{~d} y=\frac{2 a^{3}}{105}(2 a+7 \tau)
$$

and satisfies $Q \geq Q_{\min }$, where $Q_{\min }(<0)$ is given by

$$
Q_{\min }=-\frac{3087 \tau^{4}}{5120}
$$

Note that $Q=0$ when $a=a_{0}=-7 \tau / 2$, and $Q=Q_{\min }$ when $a=a_{\min }=-21 \tau / 8$. Figure 2 of Wilson \& Duffy (2005b) shows a sketch of $Q$ as a function of $a$ for $\tau>0$, $\tau=0$ and $\tau<0$. Wilson \& Duffy (2005b) also categorised and analysed all of the possible flow patterns and showed that when $\tau \geq 0$ the flow is always downwards, but that when $\tau<0$ the flow is always upwards near the contact line but, depending on the size of $a$, can be either upwards or downwards elsewhere.

\section{(a) Rivulet with Prescribed Semi-width}

The present solution can be interpreted as a rivulet of prescribed semi-width $\bar{a}$, in which case the corresponding flux $Q$ is given explicitly by (2.2) with $a=\bar{a}$. Figure 1 shows $Q$ plotted as a function of $\tau$ for a range of values of $\bar{a}$, and shows how the straight 
$\underline{\text { PSfrag replacements }}$

$$
\begin{aligned}
& \min =-\frac{3087 \tau^{4}}{5120}(\tau<0) \\
& \bar{a}=1 \\
& \bar{a}=2 \\
& \bar{a}=3 \\
& \bar{a}=4 \\
& \bar{a}=5 \\
& \bar{a}=10 \\
& \bar{a}=15 \\
& \bar{a}=20
\end{aligned}
$$

No Solution

Figure 1. Plot of the rivulet flux $Q$ given by (2.2) with $a=\bar{a}$ as function of $\tau$ for a range of values of the prescribed semi-width $\bar{a}$. The envelope curve $Q=Q_{\min }=-3087 \tau^{4} / 5120$ for $\tau<0$ is indicated with a dashed line, and the region in which there is no solution is shaded. lines for different values of $\bar{a}$ envelope to the curve $Q=Q_{\min }$ for $\tau<0$ and to $Q=0$ for $\tau \geq 0$

\section{(b) Rivulet with Prescribed Flux}

The present solution can also be interpreted as a rivulet with prescribed flux $\bar{Q}$, in which case the corresponding semi-width(s) $a$ is/are determined by solving $Q=\bar{Q}$, 


$$
\begin{array}{r}
a \\
\tau \\
\bar{Q}=0.1 \\
\bar{Q}=0.5 \\
\bar{Q}=1 \\
\bar{Q}=5 \\
\bar{Q}=10 \\
\bar{Q}=50 \\
\bar{Q}=100 \\
\bar{Q}=1000 \\
\bar{Q}=500 \\
\bar{Q}=-100 \\
\bar{Q}=-50 \\
\bar{Q}=-10 \\
\bar{Q}=-5 \\
\bar{Q}=-1 \\
\bar{Q}=-0.5 \\
\bar{Q}=-0.1 \\
a=a_{0}=-\frac{7 \tau}{2} \\
a=a_{\min }=-\frac{21 \tau}{8}
\end{array}
$$

Figure 2. Plot of the rivulet semi-width $a$ given by solving $Q=\bar{Q}$, where $Q$ is given by (2.2), as function of $\tau$ for a range of values of the prescribed flux $\bar{Q}$. Note that in the special case $\bar{Q}=0$ then $a=a_{0}=-7 \tau / 2$. The straight line $a=a_{\min }=-21 \tau / 8$ for $\tau<0$ is indicated with a dashed line.

where $Q$ is given by (2.2). Figure 2 shows $a$ plotted as a function of $\tau$ for a range of values of $\bar{Q}$, and shows that when $\bar{Q}>0$ there is a single rivulet solution for all values of $\tau$ which is a monotonically decreasing function of $\tau$ and satisfies $a>0$ when $\tau \geq 0$ and $a>a_{0}$ when $\tau<0$. In the special case $\bar{Q}=0$ there is the single solution $a=a_{0}$ for $\tau<0$. Figure 2 also shows that when $\bar{Q}<0$ there are two solutions (the narrower 
one of which is a monotonically increasing function of $\tau$ satisfying $0<a<a_{\min }$ and the wider one of which is a monotonically decreasing function of $\tau$ satisfying $a_{\min }<a<a_{0}$ ) when $\tau<\tau_{\max }$, one solution given by $a=a_{\min }$ when $\tau=\tau_{\max }$, and no solution when $\tau_{\max }<\tau<0$, where $\tau_{\max }(<0)$ is the maximum value of $\tau$ corresponding to $\bar{Q}=Q_{\min }$ given by

$$
\tau_{\max }=-\left(-\frac{5120 \bar{Q}}{3087}\right)^{1 / 4} .
$$

In the limit of small positive flux $\bar{Q} \rightarrow 0^{+}$for $\tau>0$ and for the narrower solution in the limit of small negative flux $\bar{Q} \rightarrow 0^{-}$for $\tau<0$ the rivulet becomes narrow and shallow according to

$$
a=\left(\frac{15 \bar{Q}}{2 \tau}\right)^{1 / 3}+O\left(\frac{\bar{Q}^{2}}{\tau^{5}}\right)^{1 / 3}
$$

whereas in the limit of small positive flux $\bar{Q} \rightarrow 0^{+}$for $\tau<0$ and for the wider solution in the limit of small negative flux $\bar{Q} \rightarrow 0^{-}$for $\tau<0$ the rivulet semi-width approaches the finite value $a=a_{0}$ according to

$$
a=-\frac{7 \tau}{2}-\frac{30 \bar{Q}}{49 \tau^{3}}+O\left(\frac{\bar{Q}^{2}}{\tau^{7}}\right)
$$

In the limit of large positive flux $\bar{Q} \rightarrow \infty$ the rivulet always becomes wide and deep according to

$$
a=\left(\frac{105 \bar{Q}}{4}\right)^{1 / 4}-\frac{7 \tau}{8}+O\left(\frac{\tau^{2}}{\bar{Q}^{1 / 4}}\right) .
$$

Since there is no solution when $\bar{Q}<Q_{\min }$ there is no corresponding expression in the opposite limit of large negative flux $\bar{Q} \rightarrow-\infty$. In the limit of small shear stress $\tau \rightarrow 0$ the rivulet semi-width is a regular perturbation about the finite value in the case $\tau=0$, namely $a=(105 \bar{Q} / 4)^{1 / 4}$, and is given by (2.7). In the limit of large positive shear stress $\tau \rightarrow \infty$ the effect of the shear stress overwhelms that of gravity, and the rivulet becomes narrow and shallow according to (2.5). In the limit of large negative shear stress $\tau \rightarrow-\infty$ the situation is more complicated: for the narrower rivulet when $\bar{Q}<0$ the effect of the 
shear stress again overwhelms that of gravity, and the rivulet becomes narrow and shallow according to (2.5), whereas when $\bar{Q}>0$ and for the wider rivulet when $\bar{Q}<0$ the effects of shear stress and gravity balance each other at leading order and the rivulet becomes wide and deep according to (2.6).

\subsection{Solution for a Uniform Sheet}

The corresponding solution for the unidirectional flow of a fluid sheet of uniform thickness $H$ simply has velocity $\mathbf{u}=u(z) \mathbf{i}$, where $u=(2 H-z) z / 2+\tau z$, constant pressure $p=0$ and constant free surface profile $h=H$, and hence the volume flux down a width $2 A$ is

$$
Q=\int_{-A}^{+A} \int_{0}^{H} u(z) \mathrm{d} z \mathrm{~d} y=\frac{A H^{2}}{3}(2 H+3 \tau) .
$$

Note that $Q=0$ when $H=H_{0}=-3 \tau / 2$ for $\tau<0$.

\section{Energy of a Rivulet and of a Sheet}

\subsection{Kinetic Energy}

Non-dimensionalising energy per unit length with the characteristic kinetic energy per unit length, $\rho \beta l^{2} U^{2}$, the non-dimensional kinetic energy per unit length of a rivulet of semi-width $a$, denoted by $K_{\text {riv }}$, is given by

$$
K_{\text {riv }}=\frac{1}{2} \int_{-a}^{+a} \int_{0}^{h} u^{2}(y, z) \mathrm{d} z \mathrm{~d} y=\frac{2 a^{4}}{10395}\left(8 a^{2}+55 \tau a+99 \tau^{2}\right),
$$

and the corresponding expression for the non-dimensional kinetic energy per unit length of a width $2 A$ of a sheet with uniform thickness $H$, denoted by $K_{\text {sheet }}$, is given by

$$
K_{\text {sheet }}=\frac{1}{2} \int_{-A}^{+A} \int_{0}^{H} u^{2}(z) \mathrm{d} z \mathrm{~d} y=\frac{A H^{3}}{60}\left(8 H^{2}+25 \tau H+20 \tau^{2}\right) .
$$

\subsection{Surface Energy}

Reverting momentarily for clarity to dimensional variables, the dimensional surface energy per unit length of a rivulet of semi-width $a$ on a substrate of width $2 A$, where 
necessarily $A \geq a$, is given by

$$
\gamma \int_{-a}^{+a}\left(1+h^{\prime 2}\right)^{1 / 2} \mathrm{~d} y+2 a \gamma_{L S}+2(A-a) \gamma_{V S},
$$

where $\gamma_{L S}$ and $\gamma_{V S}$ denote the surface tensions associated with the liquid-substrate and vapour-substrate interfaces, respectively, which are related to $\gamma$ and $\beta$ by the YoungDuprés equation $\gamma_{V S}=\gamma_{L S}+\gamma \cos \beta$. Using the Young-Duprés equation to eliminate $\gamma_{L S}$ and recalling that the rivulet is thin, the dimensional surface energy per unit length of a rivulet is given by

$$
\gamma\left[\frac{1}{2} \int_{-a}^{+a} h^{\prime 2} \mathrm{~d} y+a \beta^{2}\right]+2 A \gamma_{V S}
$$

Thus the non-dimensional surface energy per unit length of the rivulet, denoted by $S_{\text {riv }}$, is given by

$$
S_{\text {riv }}=\frac{1}{W}\left[\frac{1}{2} \int_{-a}^{+a} h^{\prime 2} \mathrm{~d} y+a\right]+\frac{2 A}{\hat{W}}=\frac{4 a}{3 W}+\frac{2 A}{\hat{W}},
$$

and the corresponding expression for the surface energy per unit length of a width $2 \mathrm{~A}$ of a sheet with uniform thickness $H$, denoted by $S_{\text {sheet }}$, is given by

$$
S_{\text {sheet }}=\frac{A}{W}+\frac{2 A}{W},
$$

where $W=\rho l U^{2} / \gamma \beta$ is a non-dimensional Weber number and $\hat{W}=\gamma \beta^{2} W / \gamma_{V S}=$ $\rho \beta l U^{2} / \gamma_{V S}$ is a modified non-dimensional Weber number. Note that when we consider differences between the surface energies of comparable states the term due to the surface energy of the dry substrate in (3.5) and (3.6), namely $2 A / \hat{W}$, will cancel out, and thus $\hat{W}$ will be removed from the problem.

\subsection{Total Energy}

Following previous authors we take the total energy of each state, denoted by $E_{\text {riv }}$ and $E_{\text {sheet }}$, respectively, to be the sum of their respective kinetic and surface energies, i.e. $E_{\text {riv }}=K_{\text {riv }}+S_{\text {riv }}$ and $E_{\text {sheet }}=K_{\text {sheet }}+S_{\text {sheet }}$. These expressions for $E_{\text {riv }}$ and $E_{\text {sheet }}$ 
coincide with the appropriate limits and/or special cases of the corresponding expressions obtained by previous authors, including Mikielewicz \& Moszynski (1976). However, there are two differences worth noting. Specifically, because Schmuki \& Laso (1990) (who studied the special case of purely gravity-driven flow) used only an approximate expression for the kinetic energy, they have a slightly different numerical coefficient of $a^{6}$ (namely, $4 / 3675 \simeq 0.001088$ instead of $16 / 10395 \simeq 0.001539)$ in the thin-film limit of their expression for $K_{\text {riv }}$. Similarly, because Myers, Liang \& Wetton (2004) erroneously omitted the contribution due to the surface energy of the free surface represented by the integral term in (3.5), they have the wrong numerical coefficient for $a$ (namely, 1 instead of 4/3) in the thin-film limit of their expression for $S_{\text {riv }}$.

In order to simplify the subsequent numerical calculations we can remove $W$ explicitly from the problem by rescaling the variables as follows:

$$
\begin{gathered}
a=W^{-1 / 5} a^{*}, \quad \tau=W^{-1 / 5} \tau^{*}, \quad H=W^{-1 / 5} H^{*}, \\
A=W^{-1 / 5} A^{*}, \quad Q=W^{-4 / 5} Q^{*} \\
E_{\text {riv }}=W^{-6 / 5} E_{\text {riv }}^{*}, \quad E_{\text {sheet }}=W^{-6 / 5} E_{\text {sheet }}^{*}
\end{gathered}
$$

which simply corresponds to replacing the original variables with rescaled variables and setting $W=1$ in the original problem. Hence we will, without loss of generality, take $W=1$ in all of the subsequent numerical calculations, but for clarity retain $W$ in all of the subsequent analytical results

\section{The Break-Up of a Sheet into Rivulets}

In this section we determine whether or not it is energetically favourable for a sheet to break up into a periodic array of infinitely many identical rivulets, and, when rivulets are the energetically favourable state, determine which is the most energetically favourable 
rivulet state. In order to do this we consider the difference between the energy per unit length of a section of a sheet of uniform thickness $H$ of width $2 A$ and the energy per unit length of a rivulet of semi-width $a$ on a section of the substrate of width $2 A$ with the same flux as that of the section of the sheet, denoted by $\Delta E$. Note that the possibility of a sheet breaking up into a periodic array of non-identical rivulets remains an open question.

Equating the appropriate expressions for the (non-zero) flux in the rivulet and in the sheet, given by (2.2) and (2.8), respectively, yields

$$
A=\frac{2 a^{3}(2 a+7 \tau)}{35 H^{2}(2 H+3 \tau)}
$$

while using the appropriate expressions for the energy of the rivulet and of the sheet, given by (3.1) and (3.5) and (3.2) and (3.6), respectively, yields

$$
\Delta E=\frac{A H^{3}}{60}\left(8 H^{2}+25 \tau H+20 \tau^{2}\right)+\frac{A}{W}-\left[\frac{2 a^{4}}{10395}\left(8 a^{2}+55 \tau a+99 \tau^{2}\right)+\frac{4 a}{3 W}\right] .
$$

Clearly for this situation to be physically realisable we require that the width of each rivulet does not exceed the width of the section of the sheet from which it came, i.e. that $A \geq a$, and this requirement means that not all positive values of the rivulet semi-width $a$ correspond to physically realisable rivulets. When $Q>0$ (i.e. when $\tau \geq 0$ and when $H>$ $H_{0}$ for $\tau<0$ ) then $A \geq a$ only for $a \geq a_{\text {root }}$, where $a=a_{\text {root }}$ (satisfying $a_{\text {root }}>0$ when $\tau \geq 0$ and $a_{\text {root }}>a_{0}$ when $\left.\tau<0\right)$ is the unique positive root of the cubic equation $A=a$. However, when $Q<0$ (i.e. when $0<H<H_{0}$ for $\tau<0$ ) then $A \geq a$ is possible only when $0<H \leq-H_{1} \tau$ or when $-H_{2} \tau \leq H<H_{0}$, where $H_{1} \simeq 0.6556$ and $H_{2} \simeq 1.2776$ are the two positive roots of the cubic equation $270 H^{3}-405 H^{2}+98=0$ which arises from the critical conditions $A=a$ and $\partial A / \partial a=1$, and then only for $a_{\text {root1 }} \leq a \leq a_{\text {root2 }}$, where $a=a_{\text {root1 }}$ and $a=a_{\text {root2 }}$ (satisfying $0<a_{\text {root1 }} \leq-7 \tau / 3 \leq a_{\text {root2 }}<a_{0}$ ) are the two positive roots of the cubic equation $A=a$. In particular, this means that when 
$\tau<0$ a sheet whose thickness lies in the interval $-H_{1} \tau<H<-H_{2} \tau$ is the energetically favourable state by default since there are no physically realisable rivulets into which it can break up.

(a) The Energetically Favourable State

In order to determine if it is energetically favourable for the sheet to break up into rivulets we have to determine the sign of $\Delta E$ for all physically realisable values of $a$ : if $\Delta E<0$ for all physically realisable values of $a$ then the sheet is the energetically favourable state, but if there are any physically realisable values of $a$ for which $\Delta E>0$ then rivulets are the energetically favourable state.

In the special case of zero flux, $Q=0$, in which $H=H_{0}=-3 \tau / 2$ and $a=a_{0}=-7 \tau / 2$ for $\tau<0$, it is straightforward to show that $\Delta E>0$ for all $\tau<0$, and hence that rivulets are always the energetically favourable state.

In the general case of non-zero flux, $Q \neq 0$, substituting the expression for $A$ given by (4.1) into the expression for $\Delta E$ given by (4.2) reveals that $\Delta E \sim-4 a / 3 W<0$ as $a \rightarrow 0^{+}$and $\Delta E \sim-16 a^{6} / 10385<0$ as $a \rightarrow \infty$, and hence if $\Delta E$ becomes positive then it must do so via the critical condition

$$
\Delta E=0 \quad \text { and } \quad \frac{\partial \Delta E}{\partial a}=0
$$

In practice, the critical condition (4.3) almost always occurs at a physically realisable value of $a$; when it does not the appropriate critical condition is

$$
\Delta E=0 \text { and } A=a .
$$

The only critical solution that simultaneously satisfies both (4.3) and (4.4) (i.e. that satisfies $\Delta E=0, \partial \Delta E / \partial a=0$ and $A=a)$ is

$$
\tau \simeq-2.3949 W^{-1 / 5}, \quad H \simeq 3.0622 W^{-1 / 5}, \quad a \simeq 5.4136 W^{-1 / 5}
$$


PSfrag replacements $H=-H_{1} \tau$ $\simeq-0.6556 \tau$

$H \simeq-1.5668 \tau$ $\mathrm{H}=-\mathrm{H}_{2} \tau$ $\simeq-1.2776 \tau$

$H \simeq 1.1787 \tau^{-\frac{2}{3}}$

$H \simeq 1.1787(-\tau)^{-\frac{2}{3}}$

$H=H_{0}=-\frac{3 \tau}{2}$

$H$

$\tau$

$R$

$S$

$(-2.4105,3.0798)$

$(-2.3949,3.0622)$

$(0,1.1834)$

$(-2.1622,0.9746)$

Figure 3. Plot of the $\tau-H$ parameter plane showing how it is divided by the critical curves (denoted by solid lines) into regions in which either the sheet is or rivulets are the energetically favourable state, denoted by " $S$ " and " $R$ ", respectively. The straight lines $H=-H_{1} \tau$, $H=-H_{2} \tau$ and $H=H_{0}=-3 \tau / 2$ for $\tau<0$ are shown with dashed lines, while the leading-order asymptotic solutions for large $|\tau|$ given by (4.8) and (4.9) are shown with dotted lines. The region $-H_{1} \tau<H<-H_{2} \tau$ for $\tau<0$ in which the sheet is the energetically favourable state by default since there are no physically realisable rivulets is shaded. 
$H \simeq-1.5668 \tau$

$H=-H_{1} \tau$

$\simeq-0.6556 \tau$

$\mathrm{H}=-\mathrm{H}_{2} \tau$

$\simeq-1.2776 \tau$

$H=H_{0}=-\frac{3 \tau}{2}$

H

$\Delta E=0, \frac{\partial \Delta E}{\partial a}=0$

$\Delta E=0, A=a$

$\frac{\partial \Delta E}{\partial a}=0, A=a$

$(-3.5832,4.5781)$

$(-2.4105,3.0798)$

$(-2.3949,3.0622)$

$(0,1.1834)$

$(-2.1622,0.9746)$

FiguRE 4. Sketch of the key features of Figure 3 exaggerating the narrow region of parameter space lying just above $H=-H_{2} \tau$ for $\tau<0$ and indicating which critical condition is satisfied on the different parts of the critical curves.

The task of determining the critical solutions from (4.3) and (4.4) was accomplished numerically and analytically in various asymptotic limits and special cases. The results of these calculations are plotted in Figure 3, which shows how the $\tau-H$ parameter plane is divided by the critical curves into regions in which either the sheet is or rivulets are the energetically favourable state, denoted by " $S$ " and " $R$ ", respectively.

In the special case of purely gravity-driven flow, $\tau=0$, it is straightforward to show that rivulets are the energetically favourable state for $H<H_{\mathrm{c}}$ and the sheet is the energetically favourable state for $H>H_{\mathrm{c}}$, where $H_{\mathrm{c}}$ and the associated value of $a$ are 
given by

$$
H=H_{\mathrm{c}} \simeq 1.1834 W^{-1 / 5}, \quad a \simeq 4.1951 W^{-1 / 5},
$$

in agreement with the appropriate limit of the corresponding results obtained by Mikielewicz \& Moszynski (1976).

As Figure 3 shows, the situation when $\tau>0$ and when $H>H_{0}$ for $\tau<0$ is qualitatively the same as that when $\tau=0$, namely there is a critical value of $H$ below which rivulets are the energetically favourable state and above which the sheet is the energetically favourable state. However, as Figure 3 also shows, the situation when $H<H_{0}$ for $\tau<0$ is more complicated, with a region of parameter space (including the region $-H_{1} \tau<H<$ $-H_{2} \tau$ for $\tau<0$ in which the sheet is the energetically favourable state by default) in which an arbitrarily thin sheet will remain as a sheet, and another in which an arbitrarily thick sheet will break up into rivulets. Nevertheless, whatever the value of $\tau$, sufficiently thin sheets will always break up into rivulets while sufficiently thick sheets will always remain as sheets.

Almost all of the critical curves shown in Figure 3 correspond to the critical condition (4.3), but there is a short section of critical curve between the point $(-2.3949,3.0622)$ corresponding to the critical solution $(4.5)$ and the nearby point $(-2.4105,3.0798)$ corresponding to the critical solution satisfying (4.4) and lying on the straight line $H=-H_{2} \tau$, namely

$$
\begin{gathered}
\tau=\tau_{\text {crit }} \simeq-2.4105 W^{-1 / 5}, \quad H=-H_{2} \tau_{\text {crit }} \simeq 3.0798 W^{-1 / 5}, \\
a=-\frac{7 \tau_{\text {crit }}}{3} \simeq 5.6246 W^{-1 / 5} .
\end{gathered}
$$

In order to see this and several other features of the narrow region of parameter space lying just above $H=-H_{2} \tau$ for $\tau<0$ which are difficult to discern in Figure 3, Figure 
4 shows a sketch of the key features of Figure 3 exaggerating this region and indicating which critical condition is satisfied on the different parts of the critical curves.

In order to understand fully all of the features shown in Figures 3 and 4, it is enlightening to examine various asymptotic limits and special cases analytically.

In the limits of large positive and negative shear stress $|\tau| \rightarrow \infty$ the asymptotic behaviour of the critical curves that approach $H=0$ is

$$
H \simeq 1.1787|\tau|^{-2 / 3} W^{-1 / 3} \rightarrow 0^{+}, \quad a \simeq 5.1925|\tau|^{-2 / 3} W^{-1 / 3} \rightarrow 0^{+}
$$

the former of which is included in Figure 3 and shows good agreement with the critical curves even for relatively small values of $|\tau|$. In the limit of large negative shear stress $\tau \rightarrow-\infty$ the asymptotic behaviour of the critical curve that extends to infinity is

$$
H \simeq-1.5668 \tau\left(>H_{0}\right) \rightarrow \infty, \quad a \simeq-4.1343 \tau\left(>a_{0}\right) \rightarrow \infty
$$

the former of which is also included in Figure 3 and also shows good agreement with the critical curve even for relatively small values of $-\tau$. In the limit of small shear stress $\tau \rightarrow 0$ the critical curve passing through the critical value in the special case $\tau=0$ given by (4.6) is given by $H \simeq 1.1834 W^{-1 / 5}-0.5157 \tau+O\left(\tau^{2}\right)$ and $a \simeq 4.1951 W^{-1 / 5}-1.2708 \tau+O\left(\tau^{2}\right)$, while in the limit of small negative shear stress $\tau \rightarrow 0^{-}$the two critical curves going into the origin are given by $H \sim-H_{\mathrm{c} 1} \tau \rightarrow 0^{+}$and $H \sim-H_{\mathrm{c} 2} \tau \rightarrow 0^{+}$, where $H_{\mathrm{c} 1} \simeq 0.5297$ $\left(<H_{1}\right)$ and $H_{\mathrm{c} 2} \simeq 1.3508\left(>H_{2}\right)$ are the two positive roots of the cubic equation $180 H^{3}-270 H^{2}+49=0$, and $a \sim-7 \tau / 3 \rightarrow 0^{+}$. In particular, these latter results show that the critical curves going into the origin lie strictly within the regions $0<H<-H_{1} \tau$ and $-H_{2} \tau<H<H_{0}$ as $\tau \rightarrow 0^{-}$, as sketched in Figure 4 .

On the straight line $H=-H_{1} \tau$ for $\tau<0$ the only physically realisable rivulet has semiwidth $a=a_{\text {root } 1}=a_{\text {root } 2}=-7 \tau / 3$ and so it is straightforward to show that $\Delta E<0$ for all $\tau<0$ (i.e. the sheet is always the energetically favourable state). On the other 


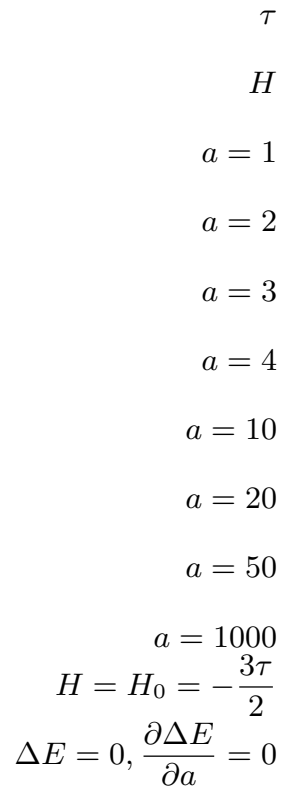

Figure 5. Contour plot of the semi-width of the most energetically favourable rivulet state in the region lying within $H>0$ for $\tau \geq 0$ and $H>H_{0}$ for $\tau<0$ in which rivulets (with $Q>0$ ) are the energetically favourable state.

hand, on the straight line $H=-H_{2} \tau$ for $\tau<0$ it can readily be shown that $\Delta E<0$ for $\tau_{\text {crit }}<\tau<0$ (i.e. the sheet is the energetically favourable state) and $\Delta E>0$ for $\tau<\tau_{\text {crit }}$ (i.e. rivulets are the energetically favourable state), where $\tau_{\text {crit }} \simeq-2.4105 W^{-1 / 5}$ is the critical value of $\tau$ satisfying (4.4) and lying on the straight line $H=-H_{2} \tau$ given by (4.7). Similar arguments show that rivulets are always the energetically favourable state in the limit $H \rightarrow H_{0}$ for $\tau<0$ and on the straight line $H \simeq-1.5668 \tau$ for $\tau<0$. 
$\tau=0$

$\tau=1$

$\tau=10$

$\tau=20$

$\tau=100$

$\tau=-9$

$\tau=-1$

$\tau=-2$

$\tau=-3$

$\tau=-4$

$\Delta E=0, \frac{\partial \Delta E}{\partial a}=0$

Figure 6. Plot of the semi-width of the most energetically favourable rivulet state as a function of $H$ for various values of $\tau$ in the region lying within $H>0$ for $\tau \geq 0$ and $H>H_{0}$ for $\tau<0$ in which rivulets (with $Q>0$ ) are the energetically favourable state. The vertical asymptotes $H=H_{0}$ of the curves corresponding to $\tau<0$ are shown with dashed lines.

(b) The Most Energetically Favourable Rivulet State

In situations in which it is energetically favourable for the sheet to break up into rivulets we can find the most energetically favourable rivulet state by determining the physically realisable rivulet state with the largest (positive) value of $\Delta E$. This will occur either when $\partial \Delta E / \partial a=0$ or when $A=a$. In what follows it is convenient to consider the regions of $\tau-H$ parameter space in which rivulets are the energetically favourable state (i.e. the regions labelled " $R$ " in Figures 3 and 4) in three separate parts.

Figures 5 and 6 show a contour plot of the semi-width of the most energetically favourable rivulet state and a plot of the same quantity as a function of $H$ for various values of $\tau$, respectively, in the region lying within $H>0$ for $\tau \geq 0$ and $H>H_{0}$ for 


\section{$\underline{\text { PSfrag replacements }}$}

$\begin{array}{r}H \\ a\end{array}$
$a=2$
$a=3$
$a=4$
$a=14$
$a=15$
$H=H_{0}=-\frac{3 \tau}{2}$

$H=-H_{2} \tau \simeq-1.2776 \tau$

$(-2.4105,3.0798)$

Figure 7. Contour plot of the semi-width of the most energetically favourable rivulet state in the region lying within $-H_{2} \tau<H<H_{0}$ for $\tau<0$ in which rivulets (with $Q<0$ ) are the energetically favourable state.

$\tau<0$ in which rivulets (with $Q>0$ ) are the energetically favourable state. In particular, Figure 6 shows that within this region the semi-width of the most energetically favourable rivulet state is a monotonically decreasing function of $H$. Specifically, it satisfies

$$
a \sim\left(\frac{33}{2 H^{2} \tau W}\right)^{1 / 2} \rightarrow \infty
$$

as $H \rightarrow 0^{+}$for $\tau>0$,

$$
a \sim\left(\frac{99}{4 H^{3} W}\right)^{1 / 2} \rightarrow \infty
$$

as $H \rightarrow 0^{+}$for $\tau=0$, and

$$
a \sim\left[\left(\frac{9(-\tau)^{5}}{320}+\frac{1}{W}\right) \frac{11}{\tau^{2}\left(H-H_{0}\right)}\right]^{1 / 2} \rightarrow \infty
$$




$$
\begin{array}{r}
{ }^{H} \\
\tau=-1 \\
\tau=-2 \\
\tau=-3 \\
\tau=-4 \\
\tau=-9 \\
\tau=-10 \\
\tau=-7 \\
\tau=-8 \\
a=\frac{7 H}{4}
\end{array}
$$

Figure 8. Plot of the semi-width of the most energetically favourable rivulet state as a function of $H$ for various values of $\tau<0$ in the region lying within $-H_{2} \tau<H<H_{0}$ for $\tau<0$ in which rivulets (with $Q<0$ ) are the energetically favourable state. The straight line $a=7 H / 4$ corresponding to $a=a_{\min }=-21 \tau / 8$ and $H=H_{0}=-3 \tau / 2$ is shown with a dashed line.

as $H \rightarrow H_{0}^{+}$for $\tau<0$, and decreases monotonically to its appropriate critical value on the critical curve.

Figures 7 and 8 and Figures 9 and 10 give the corresponding results in the regions lying within $-H_{2} \tau<H<H_{0}$ for $\tau<0$ and $0<H<-H_{1} \tau$ for $\tau<0$, respectively, in which rivulets (with $Q<0$ ) are the energetically favourable state.

Figures 7 and 8 show that within $-H_{2} \tau<H<H_{0}$ for $\tau<0$ the semi-width of the most energetically favourable rivulet state is a monotonically increasing function of $H$. Specifically, it increases monotonically from either its appropriate critical value for 
$a=1$

$a=2$

$a=3$

$a=4$

$a=5$

$a=6$

$a=10$

$a=15$

$a=20$

$\Delta E=0, \frac{\partial \Delta \stackrel{a}{E}=25}{\partial a}=0$

$(-2.1622,0.9746)$

$(-2.8170,0.7326)$

Figure 9. Contour plot of the semi-width of the most energetically favourable rivulet state in the region lying within $0<H<-H_{1} \tau$ for $\tau<0$ in which rivulets (with $Q<0$ ) are the energetically favourable state.

$\tau_{\text {crit }}<\tau<0$ or $a=-7 \tau / 3$ for $\tau<\tau_{\text {crit }}$, and satisfies $a \rightarrow a_{\text {min }}^{-}=-21 \tau / 8^{-}$(not $a \rightarrow a_{0}=-7 \tau / 2$, as naively might have been expected) as $H \rightarrow H_{0}^{-}$. To illustrate this latter result the straight line $a=7 H / 4$ obtained by eliminating $\tau$ between $a=a_{\text {min }}$ and $H=H_{0}$ is also included in Figure 8.

In the special case $H=H_{0}$ corresponding to $Q=0$ the rivulet state with semi-width $a=a_{0}$ is the only physically possible rivulet state and is therefore by default also the most energetically favourable one.

Figures 9 and 10 show that within $0<H<-H_{1} \tau$ for $\tau<0$ the semi-width of the most energetically favourable rivulet state is a monotonically decreasing function of $H$ with a discontinuous jump downwards if the location of the global maximum of 


$$
\begin{array}{r}
{ }^{H} \\
a \\
\tau=-1 \\
\tau=-2 \\
\tau=-3 \\
\tau=-4 \\
\tau=-5 \\
\tau=-10
\end{array}
$$

Figure 10. Plot of the semi-width of the most energetically favourable rivulet state as a function of $H$ for various values of $\tau<0$ in the region lying within $0<H<-H_{1} \tau$ for $\tau<0$ in which rivulets (with $Q<0$ ) are the energetically favourable state. Note that discontinuous downwards jumps in $a$ (marked with a dotted line) are possible only when $\tau<-2.8170 W^{-1 / 5}$, and hence there are no jumps in the curves corresponding to $\tau=-W^{-1 / 5}=-1$ and $\tau=-2 W^{-1 / 5}=-2$. $\Delta E$ jumps between two local maxima. Specifically, it satisfies $a \rightarrow a_{\text {min }}^{-}$as $H \rightarrow 0^{+}$ and decreases monotonically to its appropriate critical value on the critical curve. A discontinuous jump is possible only if $\Delta E$ has two local maxima and this can occur only when $\tau<-2.8170 W^{-1 / 5}$, where the critical value corresponds to the critical solution satisfying (4.4) and $\partial^{2} \Delta E / \partial a^{2}=0$, namely

$$
\tau \simeq-2.8170 W^{-1 / 5}, \quad H \simeq 0.7326 W^{-1 / 5} \quad a \simeq 4.7408 W^{-1 / 5},
$$

at which $\Delta E$ evaluated on the critical curve has a point of inflection. In particular, this 
explains why there are no jumps in the curves corresponding to $\tau=-W^{-1 / 5}=-1$ and $\tau=-2 W^{-1 / 5}=-2$ in Figure 10.

\section{The Break-Up of a Rivulet into Sub-Rivulets}

In this section we determine whether or not it is energetically favourable for a single rivulet to break up into $n(n=1,2,3, \ldots)$ identical sub-rivulets, and, when more than one sub-rivulet state is energetically favourable, determine which is the most energetically favourable sub-rivulet state. In order to do this we consider the difference between the energy of a rivulet with semi-width $a$ and the total energy of $n$ identical sub-rivulets each with semi-width $a_{n}$ with the same total flux as the original rivulet, denoted by $\Delta E_{n}$. Note that, apart from the special case of a rivulet breaking up into two sub-rivulets (in which Wilson \& Duffy (2005b) showed that the most energetically favourable state is always that of two identical sub-rivulets), the possibility of a rivulet breaking up into non-identical sub-rivulets remains an open question.

Equating the appropriate expressions for the flux in the original rivulet and in the $n$ $(n=1,2,3, \ldots)$ sub-rivulets, both given by $(2.2)$, yields

$$
a^{3}(2 a+7 \tau)=n a_{n}^{3}\left(2 a_{n}+7 \tau\right)
$$

while using the appropriate expressions for the energy of the original rivulet and of $n$ sub-rivulets, both given by (3.1) and (3.5), yields

$$
\Delta E_{n}=\frac{2 a^{4}}{10395}\left(8 a^{2}+55 \tau a+99 \tau^{2}\right)+\frac{4 a}{3 W}-n\left[\frac{2 a_{n}^{4}}{10395}\left(8 a_{n}^{2}+55 \tau a_{n}+99 \tau^{2}\right)+\frac{4 a_{n}}{3 W}\right] .
$$

The special case $n=1$, corresponding to the possibility of the "break-up" of a rivulet into a single rivulet with the same flux but a different semi-width first discussed by Wilson \& Duffy (2005b), can be readily understood. As described in Section 2, when $\tau<0$ and $a<a_{0}$ (i.e. when $Q_{\min }<Q<0$ ) there are always two rivulets with the 
same (negative) flux but different semi-widths, namely a "wider" one whose semi-width satisfies $a_{\min }<a<a_{0}$ and a "narrower" one whose semi-width satisfies $0<a<a_{\min }$. Hence, since $E_{\text {riv }}$ is a monotonically increasing function of $a$, it is always energetically favourable for a "wider" rivulet to "break up" into the corresponding "narrower" rivulet, but it is never energetically favourable for a "narrower" rivulet to "break up" into the corresponding "wider" rivulet.

\subsection{The Energetically Favourable State}

In order to determine if it is energetically favourable for the original rivulet to break up into $n$ sub-rivulets we have to determine the sign of $\Delta E_{n}$ for $n=2,3,4, \ldots$ if $\Delta E_{n}<0$ then the original rivulet is the energetically favourable state, but if $\Delta E_{n}>0$ then $n$ sub-rivulets is the energetically favourable state.

In the special case of zero flux, $Q=0$, in which $a=a_{n}=a_{0}=-7 \tau / 2$ for $\tau<0$, it is straightforward to show that $\Delta E_{n}<0$ for all $n=2,3,4, \ldots$, and hence that the original rivulet is always the energetically favourable state.

In the general case of non-zero flux, $Q \neq 0$, equations (5.1) and (5.2) reveal that $\Delta E_{n} \sim 4 a\left(1-n^{2 / 3}\right) / 3 W<0$ as $a \rightarrow 0^{+}$and $\Delta E_{n} \sim 16 a^{6}\left(1-n^{-1 / 2}\right) / 10395>0$ as $a \rightarrow \infty$, and hence that the critical condition for $\Delta E_{n}$ to become positive is simply $\Delta E_{n}=0$.

As in Section 4, the task of determining the critical solutions was accomplished numerically and analytically in various asymptotic limits and special cases. In order to do this it is, in general, convenient to write $a_{n}$ in terms of $a$ by introducing the parameter $k_{n}(>0)$ according to $a_{n}=k_{n} a$, in which case (5.1) yields an explicit expression for $a$ in terms of $\tau, n$ and $k_{n}$, namely,

$$
a=-\frac{7 \tau\left(1-n k_{n}^{3}\right)}{2\left(1-n k_{n}^{4}\right)},
$$




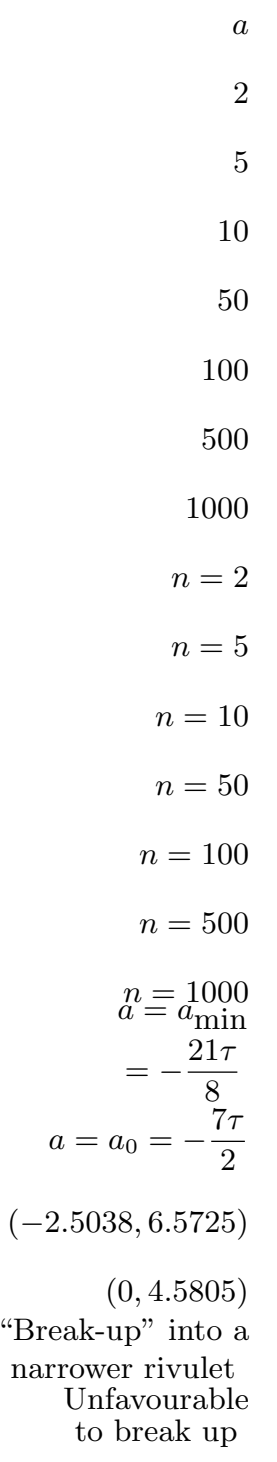

FiguRE 11. Plot of the $\tau-a$ parameter plane divided by the critical curves on which $\Delta E_{n}=0$ for $n=2,3,4, \ldots$ into regions in which either the original rivulet or $n$ sub-rivulets is the energetically favourable state. Note that when $a_{\min }<a<a_{0}$ for $\tau<0$ it is always energetically favourable for the original rivulet to "break up" into a narrower rivulet with the same flux. For clarity, the regions in which it is energetically unfavourable for the original rivulet to break up are shaded. 
and so substituting for $a$ and $a_{n}=k_{n} a$ into (5.2) yields an expression for $\Delta E_{n}$ in terms of $\tau, n, W$ and $k_{n}$ (not given here for brevity). The procedure is then to use this expression to solve the critical condition $\Delta E_{n}=0$ for $k_{n}$, and then to calculate the corresponding values of $a$ and $a_{n}=k_{n} a$ from (5.3). The results of these calculations are plotted in Figure 11, which shows how the $\tau-a$ parameter plane is divided by the critical curves on which $\Delta E_{n}=0$ for $n=2,3,4, \ldots$, denoted by $a=a_{\mathrm{c} n}$, into regions in which either the original rivulet or $n$ sub-rivulets is the energetically favourable state.

In the special case of purely gravity-driven flow, $\tau=0$, the original rivulet is the energetically favourable state for $a<a_{\mathrm{c} n}$ and $n$ sub-rivulets is the energetically favourable state for $a>a_{\mathrm{c} n}$, where $a_{\mathrm{c} n}$ and the associated value of $k_{n}$ are given by

$$
a_{\mathrm{c} n}=\left[\frac{3465\left(n^{3 / 4}-1\right)}{4\left(1-n^{-1 / 2}\right) W}\right]^{1 / 5}, \quad k_{n}=n^{-1 / 4},
$$

in agreement with the corresponding result given by equation (8) of Wilson \& Duffy (2005b) in the special case $n=2$. Evidently $a_{\mathrm{c} n}$ given by (5.4) is a monotonically increasing function of $n$, and so states with more sub-rivulets progressively become energetically favourable compared to the original rivulet as a increases. Specifically, the original rivulet is the energetically favourable state for $0<a<a_{\mathrm{c} 2}$, the two-sub-rivulet state is energetically favourable compared to the original rivulet for $a_{\mathrm{c} 2}<a<a_{\mathrm{c} 3}$, both the two- and the three-sub-rivulet states are energetically favourable compared to the original rivulet for $a_{\mathrm{c} 3}<a<a_{\mathrm{c} 4}$, and so on.

As Figure 11 shows, the situations when $\tau>0$ and when $a>a_{0}$ for $\tau<0$ are qualitatively the same as that when $\tau=0$, with the critical curves on which $\Delta E_{n+1}=0$ always lying above those on which $\Delta E_{n}=0$ for $n=2,3,4, \ldots$, and so states with more sub-rivulets progressively become energetically favourable compared to the original rivulet as $a$ increases for a given value of $\tau$. As Figure 11 also shows, the situation when $a<a_{0}$ for $\tau<0$ is complicated by the presence of the region $a_{\min }<a<a_{0}$ in 
which it is always energetically favourable for the original rivulet to "break up" into a narrower rivulet with the same flux. Nevertheless, whatever the value of $\tau$, a sufficiently narrow rivulet will always remain as a single rivulet while a sufficiently wide rivulet will always break up into sub-rivulets. Note that the results shown in Figure 11 confirm the corresponding results given by Figure 4 of Wilson \& Duffy (2005b) in the special case $n=2$. Specifically, the critical curves on which $\Delta E_{2}=0$ outwith the region $a_{\min }<a<$ $a_{0}$ agree with those given by Wilson \& Duffy (2005b). Moreover, since the critical curves on which $\Delta E_{n}=0$ for $n=3,4,5, \ldots$ (obtained here for the first time) lie above that on which $\Delta E_{2}=0$, the region that Wilson \& Duffy (2005b) identified as "unfavourable to split" is indeed the region in which the original rivulet is the energetically favourable state.

As with Figure 3 in Section 4, in order to understand fully all of the features shown in Figure 11, it is enlightening to examine various asymptotic limits and special cases analytically.

Interestingly, when $Q>0$ the behaviour of the critical curves in the limit $n \rightarrow \infty$ depends on the sign of $\tau$ : for $\tau>0$ then $a_{\mathrm{c} n}=O\left(n^{1 / 7}\right) \rightarrow \infty$ and $k_{n}=O\left(n^{-2 / 7}\right) \rightarrow 0^{+}$ according to

$$
a_{\mathrm{c} n} \sim \frac{7 \tau}{2}\left(\frac{3960}{2401 \tau^{5} W}\right)^{3 / 14} n^{1 / 7}, \quad k_{n} \sim\left(\frac{3960}{2401 \tau^{5} W}\right)^{1 / 14} n^{-2 / 7},
$$

for $\tau=0$ then $a_{\mathrm{c} n}=O\left(n^{3 / 20}\right) \rightarrow \infty$ and $k_{n}=n^{-1 / 4} \rightarrow 0^{+}$according to the leading order version of (5.4), while for $\tau<0$ then $a_{\mathrm{c} n}=O\left(n^{1 / 6}\right) \rightarrow \infty$ and $k_{n}=O\left(n^{-1 / 6}\right) \rightarrow 0^{+}$ according to

$$
a_{\mathrm{Cn}} \sim-\frac{7 \tau}{2}\left[\frac{9\left(49 \tau^{5} W-1760\right)}{9604 \tau^{5} W}\right]^{1 / 6} n^{1 / 6}, \quad k_{n} \sim\left[\frac{9604 \tau^{5} W}{9\left(49 \tau^{5} W-1760\right)}\right]^{1 / 6} n^{-1 / 6} .
$$

On the other hand, when $Q<0$ the behaviour of the critical curves in the limit $n \rightarrow \infty$ 


\section{PSfrag replacements}

$$
\begin{array}{r}
\frac{a_{\mathrm{c} n}}{n^{\frac{1}{7}}} \\
\frac{1}{7}=\frac{7 \tau}{2}\left[\frac{3960}{2401 \tau^{5} W}\right]^{\frac{3}{14}} \\
n=10 \\
n=10^{2} \\
n=10^{3} \\
n=10^{10}
\end{array}
$$

FIGURE 12. Plot showing the approach of the critical curves on which $\Delta E_{n}=0$ when $Q>0$ and $\tau>0$ for $n=10,10^{2}, 10^{3}, \ldots, 10^{10}$ (shown with solid curves) to the limiting curve given by (5.5) (shown with a dashed curve) as $n \rightarrow \infty$.

is simply

$$
a_{\mathrm{c} n} \rightarrow a_{0}, \quad k_{n} \sim \frac{1760-49 \tau^{5} W}{1760 n} \rightarrow 0^{+}
$$

for $\tau<0$. Figures 12, 13 and 14 show the approach of the critical curves on which $\Delta E_{n}=0$ to the limiting curves given by (5.5), (5.6) and (5.7) for $Q>0$ and $\tau>0$, $Q>0$ and $\tau<0$ and $Q<0$, respectively, as $n \rightarrow \infty$.

In the limits of large positive and negative shear stress $|\tau| \rightarrow \infty$ the asymptotic behaviour of the critical curves that approach $a=0$ is

$$
a_{\mathrm{c} n} \sim\left[\frac{70\left(n^{2 / 3}-1\right)}{\left(1-n^{-1 / 3}\right) \tau^{2} W}\right]^{1 / 3} \rightarrow 0^{+}, \quad k_{n} \sim n^{-1 / 3},
$$




\section{PSfrag replacements}

$\frac{a_{\mathrm{c} n}}{n^{\frac{1}{6}}}$
$\frac{\tau}{2}\left[\frac{9\left(49 \tau^{5} W-1760\right)}{9604 \tau^{5} W}\right]^{\frac{1}{6}}$
$n=10$
$n=10^{2}$
$n=10^{3}$
$n=10^{10}$

FiguRE 13. Plot showing the approach of the critical curves on which $\Delta E_{n}=0$ when $Q>0$ and $\tau<0$ for $n=10,10^{2}, 10^{3}, \ldots, 10^{10}$ (shown with solid curves) to the limiting curve given by (5.6) (shown with a dashed curve) as $n \rightarrow \infty$.

in agreement with the corresponding result given by equation (9) of Wilson \& Duffy (2005b) in the special case $n=2$. In the limit of large negative shear stress $\tau \rightarrow-\infty$ the asymptotic behaviour of the critical curves that extend to infinity is $a_{\mathrm{c} n} \sim-a_{\infty}(n) \tau$, where $a_{\infty}(n)$ is a monotonically increasing function of $n$, and $k_{n}$ is a monotonically decreasing function of $n$ satisfying $a_{\infty}(2) \simeq 4.3014(>7 / 2)$ and $k_{2} \simeq 0.9296, a_{\infty}(3) \simeq$ 4.4158 and $k_{3} \simeq 0.8905$, and $a_{\infty}(n)=O\left(n^{1 / 6}\right) \rightarrow \infty$ and $k_{n}=O\left(n^{-1 / 6}\right) \rightarrow 0^{+}$according to $(5.6)$ as $n \rightarrow \infty$. In the limit of small negative shear stress $\tau \rightarrow 0^{-}$the critical curves 


$$
\begin{array}{r}
a_{\mathrm{C} n} \\
a=a_{0}=-\frac{7 \tau}{2} \\
n=10 \\
n=10^{2} \\
n=10^{3} \\
n=10^{7} \\
n=10^{8} \\
n=10^{9} \\
n=10^{10}
\end{array}
$$

Figure 14. Plot showing the approach of the critical curves on which $\Delta E_{n}=0$ when $Q<0$ for $n=10,10^{2}, 10^{3}, \ldots, 10^{10}$ (shown with solid curves) to the limiting curve $a=a_{0}$ given by (5.7) (shown with a dashed curve) as $n \rightarrow \infty$.

going into the origin are given by

$$
a_{\mathrm{c} n} \sim-\frac{7 \tau n\left(n^{2}-1\right)}{2\left(n^{3}-1\right)} \rightarrow 0^{+}, \quad k_{n} \sim n^{-1}
$$

showing that they lie strictly within the region $a_{\min }<a<a_{0}$, as shown in Figure 11.

On the straight line $a=a_{\min }$ for $\tau<0$ it is straightforward to show that $\Delta E_{n}<0$ for $\tau_{\text {crit }}<\tau<0$ (i.e. the original rivulet is the energetically favourable state) and that $\Delta E_{n}>0$ for $\tau<\tau_{\text {crit }}$ (i.e. $n$ sub-rivulets is the energetically favourable state), where $\tau_{\text {crit }}=\tau_{\min } W^{-1 / 5}$ is the critical value of $\tau$ satisfying $\Delta E_{n}=0$, where $\tau_{\min }(n)(<0)$ is a monotonically decreasing function of $n$ satisfying $\tau_{\min }(2) \simeq-2.5038, \tau_{\min }(3) \simeq-2.6718$ 
and $\tau_{\min }(n) \simeq-1.8999 n^{2 / 15} \rightarrow-\infty$ as $n \rightarrow \infty$. Similar arguments show that the original rivulet is always the energetically favourable state in the limit $a \rightarrow a_{0}$ for $\tau<0$.

\subsection{The Most Energetically Favourable Sub-Rivulet State}

In situations in which it is energetically favourable for the original rivulet to break up into sub-rivulets we can find the most energetically favourable sub-rivulet state by determining the sub-rivulet state with the largest (positive) value of $\Delta E_{n}$. The critical condition for the most favourable sub-rivulet state to jump from one state to another is when two states with different numbers of sub-rivulets share the largest value of $\Delta E_{n}$, and it is found that this always occurs when "neighbouring" sub-rivulet states with $n$ and $n+1$ rivulets have the same energy, i.e. when the difference between the energies of the two states, denoted by $\Delta E_{n, n+1}=\Delta E_{n+1}-\Delta E_{n}$, is zero. The situation is therefore rather similar to that described in Section 5.1, which involved comparing the energy of one rivulet with that of $n$ sub-rivulets, except that now it involves comparing the energy of $n$ sub-rivulets with that of $n+1$ sub-rivulets. We therefore proceed in the same manner as described in Section 5.1 with the appropriate modifications. For example, it is now convenient to write both $a_{n}$ and $a_{n+1}$ in terms of $a$ by introducing the parameters $k_{n}$ $(>0)$ and $k_{n+1}(>0)$ according to $a_{n}=k_{n} a$ and $a_{n+1}=k_{n+1} a$, in which case (5.1) yields (5.3) and

$$
a=-\frac{7 \tau\left(1-(n+1) k_{n+1}^{3}\right)}{2\left(1-(n+1) k_{n+1}^{4}\right)}=-\frac{7 \tau\left((n+1) k_{n+1}^{3}-n k_{n}^{3}\right)}{2\left((n+1) k_{n+1}^{4}-n k_{n}^{4}\right)},
$$

and so substituting for $a, a_{n}=k_{n} a$ and $a_{n+1}=k_{n+1} a$ into (5.2) yields an expression for $\Delta E_{n, n+1}$ which is used to solve the critical condition $\Delta E_{n, n+1}=0$ for either $k_{n}$ or $k_{n+1}$ (they are related via (5.10)) and hence obtain $a, a_{n}=k_{n} a$ and $a_{n+1}=k_{n+1} a$ from (5.10). The results of these calculations are plotted in Figure 15, which shows how the $\tau-a$ parameter plane is divided by the critical curves on which $\Delta E_{n, n+1}=0$ for 
$n=2$

$n=3$

$n=4$

$n=5$

$n=10$

$n=50$

$a \stackrel{n}{=} \overline{\bar{a}}_{\min }^{100}$

$=-\frac{21 \tau}{8}$

$a=a_{0}=-\frac{7 \tau}{2}$

$n=2$

Unfavourable

to break up

Unfavourable

to break up

"Break-up" into a

narrower rivulet

$(-2.5038,6.5725)$

$(0,4.5805)$

FIGURE 15. Plot of the $\tau-a$ parameter plane divided by the critical curves on which $\Delta E_{n, n+1}=0$ for $n=2,3,4, \ldots$ into regions in which either the original rivulet or $n$ sub-rivulets is the most energetically favourable state. Note that when $a_{\min }<a<a_{0}$ for $\tau<0$ it is always energetically favourable for the original rivulet to "break up" into a narrower rivulet with the same flux. For clarity, the regions in which it is energetically unfavourable for the original rivulet to break up are shaded. 
$n=1,2,3, \ldots$, denoted by $a=a_{\mathrm{c} n, n+1}$, into regions in which either the original rivulet or $n$ sub-rivulets is the most energetically favourable state.

In the special case of purely gravity-driven flow, $\tau=0$, the original rivulet is the most energetically favourable state for $0<a<a_{\mathrm{c} 1,2}$, the two-sub-rivulet state is the most energetically favourable for $a_{\mathrm{c} 1,2}<a<a_{\mathrm{c} 2,3}$, the three-sub-rivulet state is the most energetically favourable for $a_{\mathrm{c} 2,3}<a<a_{\mathrm{c} 3,4}$, and so on, where $a_{\mathrm{c} n, n+1}$ and the associated values of $k_{n}$ and $k_{n+1}$ are given by

$$
a_{\mathrm{c} n, n+1}=\left[\frac{3465\left\{(n+1)^{3 / 4}-n^{3 / 4}\right\}}{4\left\{(n+1)^{-1 / 2}-n^{-1 / 2}\right\} W}\right]^{1 / 5}, \quad k_{n}=n^{-1 / 4}, \quad k_{n+1}=(n+1)^{-1 / 4}
$$

As in Section 5.1, it is enlightening to examine various asymptotic limits and special cases analytically.

Unlike in Section 5.1, the behaviour of the critical curves in the limit $n \rightarrow \infty$ is independent of the sign of $\tau$, but the calculation to determine it is more complicated. In order to calculate the leading order behaviour of the critical curves $\Delta E_{n, n+1}=0$ it is necessary to expand both $k_{n}$ and $k_{n+1}$ up to and including $O\left(n^{-5 / 4}\right)$ according to

$$
\begin{gathered}
k_{n}=c_{11} n^{-1 / 4}+c_{12} n^{-1 / 2}+c_{13} n^{-3 / 4}+c_{14} n^{-1}+c_{15} n^{-5 / 4}+O\left(n^{-3 / 2}\right), \\
k_{n+1}=c_{21} n^{-1 / 4}+c_{22} n^{-1 / 2}+c_{23} n^{-3 / 4}+c_{24} n^{-1}+c_{25} n^{-5 / 4}+O\left(n^{-3 / 2}\right),
\end{gathered}
$$

and substituting these expansions into (5.10) we deduce that $c_{11}=c_{21}, c_{12}=c_{22}$, $c_{13}=c_{23}$ and $c_{14}=c_{24}$ but that

$$
c_{25}=c_{15}-\frac{c_{11}}{c_{11}^{4}+3}\left(\neq c_{15}\right)
$$

Substituting the expansions for $k_{n}$ and $k_{n+1}$ into the critical condition $\Delta E_{n, n+1}=0$ reveals that $c_{11}$ is the unique real and positive solution of

$$
49 c_{11}^{12} \tau^{5}\left(9 c_{11}^{12}-27 c_{11}^{8}-176 c_{11}^{4}-198\right)-15840\left(c_{11}^{4}+2\right)\left(c_{11}^{4}-1\right)^{5}=0,
$$




\section{PSfrag replacements}

$$
\begin{array}{r}
\frac{a_{\mathrm{C} n, n+1}}{n^{\frac{1}{4}}} \\
\frac{a_{\mathrm{C} n, n+1}}{n^{\frac{1}{4}}}=\frac{7 \tau c_{11}^{3}}{1-c_{11}^{4}} \\
(0,4.1951) \\
n=10 \\
n=10^{2} \\
n=10^{3} \\
n=10^{6}
\end{array}
$$

FIGURE 16. Plot showing the approach of the critical curves on which $\Delta E_{n, n+1}=0$ when $Q>0$ for $n=10,10^{2}, 10^{3}, \ldots, 10^{6}$ (shown with solid curves) to the limiting curve given by (5.16), where $c_{11}$ is the unique real and positive solution of (5.15) (shown with a dashed curve) as $n \rightarrow \infty$.

and hence that the critical curves become large like $O\left(n^{1 / 4}\right) \rightarrow \infty$ as $n \rightarrow \infty$ according to

$$
a_{\mathrm{c} n, n+1} \sim \frac{7 \tau c_{11}^{3}}{1-c_{11}^{4}} n^{1 / 4}, \quad k_{n} \sim k_{n+1} \sim c_{11} n^{-1 / 4} .
$$

Figure 16 shows the approach of the critical curves on which $\Delta E_{n, n+1}=0$ to the limiting curve given by (5.16) as $n \rightarrow \infty$.

In the limits of large positive and negative shear stress $|\tau| \rightarrow \infty$ the asymptotic be- 
haviour of the critical curves that approach $a=0$ is

$$
a_{\mathrm{c} n, n+1} \sim\left[\frac{70\left\{(n+1)^{2 / 3}-n^{2 / 3}\right\}}{\left\{(n+1)^{-1 / 3}-n^{-1 / 3}\right\} \tau^{2} W}\right]^{1 / 3}, \quad k_{n} \sim n^{-1 / 3}, \quad k_{n+1} \sim(n+1)^{-1 / 3},
$$

while in the limit of large negative shear stress $\tau \rightarrow-\infty$ we have $a_{\mathrm{c} n, n+1} \sim-a_{\infty}(n) \tau$, where $a_{\infty}(n)$ is a monotonically increasing function of $n$, and $k_{n}$ and $k_{n+1}$ are monotonically decreasing functions of $n$ satisfying $a_{\infty}(1) \simeq 4.3014(>7 / 2), k_{1}=1$ and $k_{2} \simeq 0.9296, a_{\infty}(2) \simeq 4.6175, k_{2} \simeq 0.9156$ and $k_{3} \simeq 0.8774$, and $a_{\infty}(n)=O\left(n^{1 / 4}\right) \rightarrow \infty$ and $k_{n} \sim k_{n+1}=O\left(n^{-1 / 4}\right) \rightarrow 0^{+}$according to (5.16) as $n \rightarrow \infty$.

Note that since $\Delta E_{1,2}=0$ is exactly the same as $\Delta E_{2}=0$, then $a_{\mathrm{c} 1,2}$ coincides with $a_{\mathrm{c} 2}$, and so, for example, setting $n=1$ in (5.11) is the same as setting $n=2$ in (5.4).

\section{Purely Shear-Stress-Driven Flow}

Although somewhat obscured by the choice of non-dimensionalisation, the present results include those for the special case of purely shear-stress-driven flow. Specifically, if we neglect gravity (i.e. set $g=0$ ) and non-dimensionalise with a characteristic transverse length scale $L$ (rather than the capillary length $\ell$ ) and the characteristic vertical velocity $U=\tau \beta L / \mu\left(\right.$ rather than $\left.U=\rho g \beta^{2} \ell^{2} / \mu\right)$ then $u$ and $Q$ are given by $u=z$ and $Q=2 a^{3} / 15$ for a rivulet and $Q=A H^{2}$ for a sheet. For the break-up of a sheet into rivulets, from (4.8) rivulets are the energetically favourable state for $H<H_{\mathrm{c}}$ and the sheet is the energetically favourable state for $H>H_{\mathrm{c}}$, where $H_{\mathrm{c}}$ and the associated value of $a$ are given by $H_{\mathrm{c}} \simeq 1.1787 W^{-1 / 3}$ and $a \simeq 5.1925 W^{-1 / 3}$. For the break-up of a rivulet into $n$ $(n=2,3,4, \ldots)$ sub-rivulets, from $(5.8)$, the original rivulet is the energetically favourable state for $0<a<a_{\mathrm{c} 2}$, the two-sub-rivulet state is energetically favourable compared to the original rivulet for $a_{\mathrm{c} 2}<a<a_{\mathrm{c} 3}$, both the two- and the three-sub-rivulet states are energetically favourable compared to the original rivulet for $a_{\mathrm{c} 3}<a<a_{\mathrm{c} 4}$, and so on, 
where $a_{\mathrm{c} n}$ and the associated value of $k_{n}$ are given by

$$
a_{\mathrm{C} n}=\left[\frac{70\left(n^{2 / 3}-1\right)}{\left(1-n^{-1 / 3}\right) W}\right]^{1 / 3}, \quad k_{n}=n^{-1 / 3}
$$

and, from (5.17), the original rivulet is the most energetically favourable state for $0<a<$ $a_{\mathrm{c} 1,2}$, the two-sub-rivulet state is the most energetically favourable for $a_{\mathrm{c} 1,2}<a<a_{\mathrm{C} 2,3}$, the three-sub-rivulet state is the most energetically favourable for $a_{\mathrm{C} 2,3}<a<a_{\mathrm{C} 3,4}$, and so on, where $a_{\mathrm{C} n, n+1}$ and the associated values of $k_{n}$ and $k_{n+1}$ are given by

$$
a_{\mathrm{c} n, n+1}=\left[\frac{70\left\{(n+1)^{2 / 3}-n^{2 / 3}\right\}}{\left\{(n+1)^{-1 / 3}-n^{-1 / 3}\right\} W}\right]^{1 / 3}, \quad k_{n}=n^{-1 / 3}, \quad k_{n+1}=(n+1)^{-1 / 3} .
$$

\section{Conclusions}

We used the lubrication approximation to obtain a complete description of the energetics of the break-up both of a fluid sheet of uniform thickness into a periodic array of infinitely many identical thin rivulets and of a single thin rivulet into one or more identical sub-rivulets on a vertical substrate in the presence of a prescribed uniform longitudinal shear stress at the free surface of the fluid by comparing the total energies of the different states.

Figure 3 (and the accompanying sketch in Figure 4) shows when it is energetically favourable for a sheet to break up into rivulets, while Figures 5-10 show the most energetically favourable rivulet state in the appropriate regions of $\tau-H$ parameter space. Correspondingly, Figure 11 shows when it is energetically favourable for a rivulet to break up into sub-rivulets, while Figure 15 shows the most energetically favourable sub-rivulet state in the appropriate regions of $\tau-a$ parameter space. For both problems the situation when $Q>0$ is relatively straightforward (and, in particular, qualitatively the same as that in the case $\tau=0$ ), but when $Q<0$ it is more complicated. However, as Figures 3 and 11 show, whatever the value of $\tau$, there is always a critical thickness below which it 
is energetically favourable for a sheet to break up into rivulets and a critical semi-width below which it is energetically favourable for a rivulet to remain as a single rivulet (although both the critical thickness and the critical semi-width approach zero like $O(-\tau)$ in the limit of small negative shear stress $\tau \rightarrow 0^{-}$), and a critical thickness above which it is energetically favourable for a sheet to remain as a sheet and a critical semi-width above which it is energetically favourable for a rivulet to break up into sub-rivulets.

While previous authors have demonstrated good agreement between predictions based on the approach used in the present work (i.e. based on comparing the energies of the different states) and experimental results for the minimum thickness of a uniform film, we are not aware of any experimental results against which the present theoretical predictions can be directly compared. Hopefully such results will become available in the future, especially in the most interesting case when $Q<0$.

The second author (JMS) gratefully acknowledges the financial support of the United Kingdom Engineering and Physical Sciences Research Council via a research studentship. All three authors gratefully acknowledge useful discussions with Dr Ian Taylor and Mr Andrew Robertson in the Department of Mechanical Engineering at the University of Strathclyde on various aspects of thin-film flow in the presence of an external airflow. 


\section{REFERENCES}

Bankoff, S. G. 1971 Minimum thickness of a draining liquid film. Int. J. Heat Mass Transfer 14, 2143-2146.

Chung, J. C. \& Bankoff, S. G. 1980 Initial breakdown of a heated liquid film in cocurrent two-component annular flow: II. rivulet and drypatch models. Chem. Eng. Commumn. 4, $455-470$.

Doniec, A. 1988 Flow of a laminar liquid film down a vertical surface. Chem. Eng. Sci. 43, $847-854$.

Doniec, A. 1991 Laminar flow of a liquid rivulet down a vertical solid surface. Can. J. Chem. Eng. 69, 198-202.

El-Genk, M. S. \& Saber, H. H. 2001 Minimum thickness of a flowing down liquid film on a vertical surface. Int. J. Heat Mass Transfer 44, 2809-2825.

El-Genk, M. S. \& SABer, H. H. 2002 An investigation of the breakup of an evaporating liquid film, falling down a vertical, uniformly heated wall. J. Heat Transfer 124, 39-50.

Hartley, D. E. \& Murgatroyd, W. 1964 Criteria for the break-up of thin liquid layers flowing isothermally over solid surfaces. Int. J. Heat Mass Transfer 7, 1003-1015.

Hobler, T. 1964 Minimum surface wetting. Chemia Stosowana 2B, 145-159 (in Polish).

Hughes, D. T. \& Botт, T. R. 1998 Minimum thickness of a liquid film flowing down a vertical tube. Int. J. Heat Mass Transfer 41, 253-260.

Mikielewicz, J. \& Moszynski, J. R. 1976 Minimum thickness of a liquid film flowing vertically down a solid surface. Int. J. Heat Mass Transfer 19, 771-776.

Mikielewicz, J. \& Moszynski, J. R. 1978 An improved analysis of breakdown of thin liquid films. Archives of Mechanics 30, 489-500.

Myers, T. G., Liang, H. X. \& Wetton, B. 2004 The stability and flow of a rivulet driven by interfacial shear and gravity. Int. J. Non-Linear Mech. 39, 1239-1249.

Robertson, A. C., Taylor, I. J., Wilson, S. K., Duffy, B. R. \& Sullivan, J. M. 2010 Numerical simulation of rivulet evolution on a horizontal cable subject to an external aerodynamic field. J. Fluids and Structures 26, 50-73. 
SABer, H. H. \& El-Genk, M. S. 2004 On the breakup of a thin liquid film subject to interfacial shear. J. Fluid Mech. 500, 113-133.

Schmuki, P. \& Laso, M. 1990 On the stability of rivulet flow. J. Fluid Mech. 215, 125-143.

Sullivan, J. M., Wilson, S. K. \& Duffy, B. R. 2008 A thin rivulet of perfectly wetting fluid subject to a longitudinal surface shear stress. Q. J. Mech. Appl. Math. 61, 25-61.

Wilson, S. K. \& Duffy, B. R. 2005a When is it energetically favourable for a rivulet of perfectly wetting fluid to split? Phys. Fluids 17, 078104.

Wilson, S. K. \& Duffy, B. R. 2005b Unidirectional flow of a thin rivulet on a vertical substrate subject to a prescribed uniform shear stress at its free surface. Phys. Fluids 17, 108105. 